Research Paper

\title{
TREM-1 promoted apoptosis and inhibited autophagy in LPS-treated HK-2 cells through the NF-KB pathway
}

Pan Pan1,\#, Xudong Liu ${ }^{3}, \#$, LingLing Wu ${ }^{4, \#}$, Xiaogang $\mathrm{Li}^{3}$, Kaifei Wang ${ }^{1}$, Xiaoting Wang ${ }^{2}$, Xiang Zhou ${ }^{2}$, Yun Long2, Dawei Liu2, Lixin Xie ${ }^{1,}$, Longxiang Su, ${ }^{2}$

1. College of Pulmonary and Critical Care Medicine, Chinese PLA General Hospital, 17th Heishanhujia, Haidian District, Beijing 100091, China

2. Department of Critical Care Medicine, Peking Union Medical College Hospital, Peking Union Medical College \& Chinese Academy of Medical Sciences, Beijing 100730, China

3. Medical Science Research Center, Peking Union Medical College Hospital, Peking Union Medical College \& Chinese Academy of Medical Sciences, Beijing 100730, China

4. Department of Nephrology, Chinese PLA General Hospital, Chinese PLA Institute of Nephrology, State Key Laboratory of Kidney Diseases, National Clinical Research Center for Kidney Diseases, Beijing 100853, China

\#Dr. Pan Pan, Xudong Liu, and Lingling Wu contributed equally to this work.

$\triangle$ Corresponding authors: Longxiang Su, Department of Critical Care Medicine, Peking Union Medical College Hospital, 1 Shuaifuyuan, Dongcheng District, Beijing 100730, China. E-mail address: sulongxiang@vip.163.com Or Lixin Xie, Center of Pulmonary and Critical Care Medicine, Chinese PLA General Hospital, 17th Heishanhujia, Haidian District, Beijing 100091, China. E-mail address: xielx301@126.com

(C) The author(s). This is an open access article distributed under the terms of the Creative Commons Attribution License (https://creativecommons.org/licenses/by/4.0/). See http://ivyspring.com/terms for full terms and conditions.

Received: 2020.07.20; Accepted: 2020.10.22; Published: 2021.01.01

\begin{abstract}
Triggering receptor expressed by myeloid cells (TREM-1) is an amplifier of inflammatory responses triggered by bacterial or fungal infection. Soluble TREM-1 (sTREM-1) expression was found to be upregulated in sepsis-associated acute kidney injury (SA-AKI) and predicted to be a potential biomarker. However, the mechanism remains unclear. The human kidney-2 (HK-2) cell line was treated with lipopolysaccharide (LPS) and used to examine the potential roles of TREM-1 in apoptosis and autophagy. A cell viability assay was employed to assess the number of viable cells and as a measure of the proliferative index. The concentrations of sTREM-1, interleukin (IL)-1 $\beta$, tumor necrosis factor- $\alpha$ (TNFa) and IL- 6 in cell-free culture supernatants were measured by enzyme-linked immunosorbent assay (ELISA). Western blot analysis was performed to analyze apoptosis, autophagy and the relevant signaling pathways. The results suggested that TREM-1 overexpression after LPS treatment decreased proliferation and increased apoptosis. The concentrations of sTREM-1, IL-1 $\beta$, TNFa and IL-6 in cell-free culture supernatants were increased in the TREM-1 overexpression group after LPS treatment. Expression of the antiapoptotic gene $\mathrm{Bcl}-2$ was downregulated in the TREM-1 overexpression group, while that of the proapoptotic genes Bax, cleaved caspase-3 and cleaved caspase- 9 was upregulated. Overexpression of TREM-1 downregulated expression of the autophagy genes Beclin-1, Atg-5 and LC3b and increased the gene expression of $\mathrm{p} 62$, which inhibits autophagy. Conversely, treatment with TREM-1-specific shRNA had the opposite effects. The nuclear factor-KB (NF-KB) signaling pathway (P-p65/p65 and P-IKBa/IKBa) in LPS-induced HK-2 cells was regulated by TREM-1. In summary, TREM-1 promoted apoptosis and inhibited autophagy in HK-2 cells in the context of LPS exposure potentially through the NF-KB pathway.
\end{abstract}

Key words: Sepsis, AKI, TREM-1, Apoptosis, Autophagy, NF-KB signaling pathway

\section{Introduction}

Sepsis is the leading cause of death in patients admitted to the intensive care unit, and death from sepsis is caused by a deleterious immune response to infection [1]. Severe sepsis is associated with multiorgan dysfunction, but the mechanisms leading to this dysfunction remain unclear [2]. The kidney is the organ most commonly affected by sepsis, leading to sepsis-associated acute kidney injury (SA-AKI). Acute kidney injury (AKI) develops in up to $60 \%$ of patients with sepsis, and up to $50 \%$ of patients with 
AKI have sepsis [3]. Earlier recognition, diagnosis and treatment can improve renal outcomes and decrease mortality [4]. According to recent studies, soluble triggering receptor expressed on myeloid cell-1 (sTREM-1) may be a relatively sensitive and accurate biomarker for the diagnosis of sepsis and infectious diseases $[5,6]$. We found that urine sTREM-1 could be used to diagnose sepsis or even provide an early warning of possible secondary AKI in sepsis patients $[7,8]$.

sTREM-1 is a 17-kDa fragment cleaved from triggering receptor expressed on myeloid cell-1 (TREM-1) by a metalloproteinase [9]. The TREM family is a recently discovered family whose members are expressed on the cell surface and play important roles in innate and adaptive immunity [10]. Among the six identified TREM proteins (TREM-1, TREM-2, and TREML1-4), TREM-1 is a transmembrane glycoprotein expressed on monocytes, neutrophils and macrophages [11-13]. TREM-1 is an amplifier of inflammatory and immune responses that functions by mediating cross-talk with TLRs and/or NLRs, which are predominantly associated with bacterial and fungal infection [14]. TREM-1 expression was found to be significantly increased in response to lipopolysaccharide (LPS), subsequently promoting the release of various proinflammatory cytokines, such as interleukins (ILs; including IL-1 $\beta$ ) and tumor necrosis factor- $\alpha$ (TNF- $\alpha$ ) [12]. Knockdown of TREM-1 expression inhibited the activity of the nuclear factor-кB (NF-kB) pathway [15]. Sepsis induced the activation of granulocytes and monocytes/macrophages, which highly express TREM-1.

The inflammatory response during AKI damages tubular epithelial cells (TECs) [16], with significant damage and necrosis observed in the proximal tubule. The proinflammatory response mediated by TEC activation was shown to activate dendritic cells, produce chemokines, and activate $\mathrm{T}$ lymphocyte cells [17]. In addition, activated TECs directly interact with neutrophils, monocytes and $\mathrm{T}$ cells [18]. TREM-1 expression was also reported to be increased in TECs after ischemia-reperfusion injury (IRI). TEC-associated TREM-1 in IRI was shown to impact cell fate and metabolism [19]. A previous study reported that sTREM-1 levels were significantly increased at the time of diagnosis and $24 \mathrm{~h}$ before AKI diagnosis $[8,20]$. We hypothesized that the local inflammatory response in the kidney contributes to the elevated secretion of urine sTREM-1 during SA-AKI [7]. However, the function of TREM-1 in SA-AKI remains unclear. In the present work, we examined the potential roles of TREM-1 in apoptosis and autophagy in the human kidney-2 (HK-2) cell line treated with LPS to determine how TREM-1 damages parenchymal cells in the kidneys.

\section{Materials and Methods}

\section{Cell culture and treatment}

HK-2 human renal proximal TECs (American Type Culture Collection, Manassas, VA, USA) were cultured in Dulbecco's modified Eagle's medium and Ham's F-12 medium (DMEM/F12, Gibco) supplemented with $10 \%$ fetal bovine serum, 100 $\mathrm{U} / \mathrm{mL}$ penicillin, and $100 \mathrm{mg} / \mathrm{mL}$ streptomycin. HK-2 cells at logarithmic growth phase were digested with trypsin (Solarbio, T1300-100), seeded in a 6-well plate $\left(3 \times 10^{5} /\right.$ well $)$, and then cultured for another $8 \mathrm{~h}$ before treatment.

For TREM-1 depletion studies, TREM-1-specific siRNA (Santa Cruz, Cat\# sc-42999), control (CON) shRNA plasmid (Santa Cruz, Cat\# sc-108060), TREM-1 cDNA ORF clone (Sino Bio, Cat\# HG10511-UT), or negative control vector (Sino Bio, Cat\# CV011) was transfected into HK-2 cells using Lipofectamine 3000 (Thermo Fisher). Cells were grown to a subconfluent density at $37^{\circ} \mathrm{C}$ with $5 \% \mathrm{CO}_{2}$ and stimulated with LPS (100 ng/mL).

\section{Cell viability assay}

The Cell Counting Kit-8 (CCK8) assay was employed to assess the number of viable cells and as a measure of the proliferation index. HK-2 cells were plated in 96-well plates at a density of $1 \times 10^{5}$ cells/well and then cultured in complete medium with or without LPS $(100 \mathrm{ng} / \mathrm{ml})$. The optical density at 450 $\mathrm{nm}$ (OD450) was determined at each time point.

\section{qRT-PCR}

Total mRNA was extracted from primary monocytes using the RNeasy ${ }^{\circledR}$ Micro Kit (QIAGEN). RNA quantity was measured with a Nanodrop ${ }^{\mathrm{TM}}$ 2000C spectrophotometer (Thermo Fisher Scientific) and reverse transcribed using iScript ${ }^{\mathrm{TM}}$ Reverse Transcription SuperMix for qRT-PCR (Bio-Rad) according to the manufacturer's instructions. The RT-PCR conditions used for all reactions were as follows: $25^{\circ} \mathrm{C}, 5 \mathrm{~min} / 46^{\circ} \mathrm{C}, 20 \mathrm{~min} / 95^{\circ} \mathrm{C}$, and $1 \mathrm{~min}$ per cycle. Reverse transcription was performed on a MyiQ thermocycler (Bio-Rad). The relative expression of TREM-1 in HK-2 cells was then analyzed by qPCR using Sso advanced Universal SYBR Green Supermix (Bio-Rad) and human TREM-1 probes (QuantiTect Primers Assay, QIAGEN). GAPDH served as a housekeeping control gene. qPCR was performed on a MyiQ thermocycler, and the results were quantified with iQ5 software (QIAGEN). The expression levels of each target gene in each sample were calculated by the comparative $\mathrm{Ct}$ method after normalization to the 
expression levels of the housekeeping gene.

\section{Enzyme-linked immunosorbent assay (ELISA)}

The concentrations of sTREM-1, IL-1 $\beta$, TNFa and IL-6 in cell-free culture supernatants were measured using commercially available specific ELISA kits (DuoSet, R\&D Systems) according to the manufacturer's instructions. The detection limits were $31.3 \mathrm{pg} / \mathrm{ml}$ (sTREM-1 and IL-6), $15.6 \mathrm{pg} / \mathrm{ml}$ (TNFa) and $3.91 \mathrm{pg} / \mathrm{ml}$ (IL-1 $\beta)$.

\section{Western blot analysis}

Western blot analysis was performed using standard procedures. The primary antibodies used were anti-TREM-1, anti-caspase 3 and anti-caspase 9 antibodies (Abcam, Cambridge, MA, USA) and anti-ATG5, anti-BECN1, anti-LC3 and anti-P62 (Santa Cruz Biotechnology, Santa Cruz, CA, USA). Horseradish peroxidase-conjugated secondary antibodies were used, and specific antibody-antigen complexes were detected using a chemiluminescent substrate.

\section{Statistical analyses}

Experiments were performed at least in triplicate, and the data are expressed as the mean \pm standard deviation (SD). For statistical analyses, the data were analyzed using Student's t-test. $P<0.05$ was used to indicate significance. One-way ANOVA was used for comparisons among different groups.

\section{Results}

\section{TREM-1 expression in HK-2 cells was induced following LPS stimulation}

RT-PCR and western blotting were performed to evaluate TREM-1 gene and protein expression, respectively, in HK-2 cells after TREM-1 vector or shRNA transfection (Figure 1). Both the gene and protein levels (protein ratio in the treated vs. control group) revealed that TREM-1 expression was significantly increased in cells transfected with the TREM-1 vector but decreased in cells transfected with TREM-1-shRNA (CON vs. TREM-1 vector vs. TREM-1-shRNA, $P<0.05)$. These results demonstrated the successful overexpression or suppression of TREM-1 in HK-2 cells. As shown in the same figure, LPS stimulated TREM-1 expression in HK-2 cells, as evidenced by increased gene and protein expression in the LPS-treated group compared to the vector and shRNA control groups (LPS gene + CON vector vs. LPS + CON shRNA vs. vector vs. shRNA group, $950.40 \pm 64.01$ vs. $1260.07 \pm 129.91$ vs. $143656.67 \pm 9916.67$ vs. $126.28 \pm 14.96$ vs. $1 \pm 0$; LPS protein vs. vector vs. shRNA group vs. control, $0.81 \pm 0.02$ vs. $0.77 \pm 0.03$, $0.54 \pm 0.03$ vs. $0.49 \pm 0.02$ vs. $0.5 \pm 0.03$, both $P<0.05$ ). TREM-1 expression was further augmented by LPS in cells transfected with the TREM-1 vector (LPS gene + CON vector group vs. LPS + TREM-1 vector group, $950.40 \pm 64.01$ vs. $608376.67 \pm 47177.31$; LPS protein vs. LPS + TREM-1 vector group $0.96 \pm 0.05$ vs. $1.19 \pm 0.15, P$ $<0.05)$ but attenuated in cells transfected with TREM-1-specific shRNA even in the presence of LPS (LPS gene + CON shRNA group vs. LPS + TREM-1 shRNA group, $1260.07 \pm 129.91$ vs. $45.35 \pm 7.37$; LPS protein vs. LPS + TREM-1 shRNA group, $0.77 \pm 0.03$ vs. $0.32 \pm 0.03, P<0.05)$.

\section{Cell viability and apoptosis in response to LPS and TREM- 1 intervention}

As shown in Figure 2A, HK-2 cell viability was decreased in response to LPS treatment (CON vector vs. $\mathrm{CON}$ vector+LPS, $1.26 \pm 0.02$ vs. $0.77 \pm 0.02$; $\mathrm{CON}$
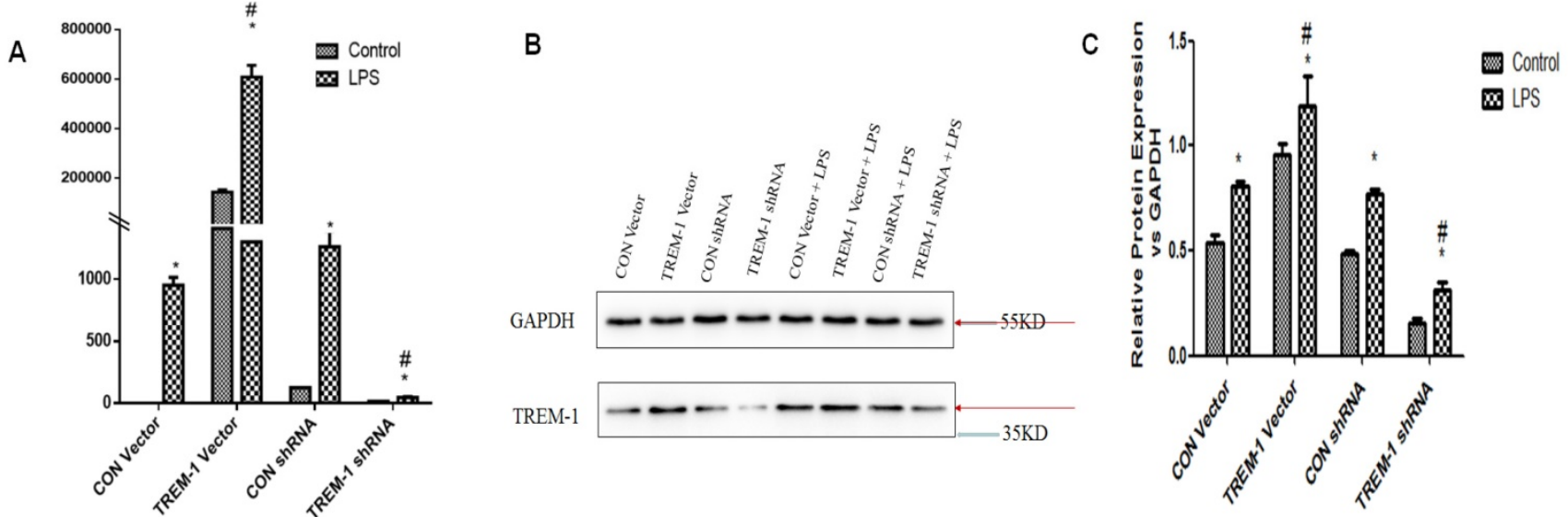

Figure 1. TREM-1 expression in HK-2 cells treated with a TREM-1 vector or shRNA with or without LPS. HK-2 cells were transfected with a control vector, plasmid for TREM-1 expression, control siRNA or TREM-1-specific siRNA as described in the Materials and Methods section. Panel A: Representative PT-PCR results indicating suppression of TREM-1 by siRNA (TREM-1 siRNA) or overexpression of TREM-1 (TREM-1 DNA). Panels B \& C: Representative immunoblotting results indicating suppression of TREM-1 by siRNA (TREM-1 siRNA) or overexpression of TREM-1 (TREM-1 DNA) from qualitative or quantitative analysis, respectively. $* P<0.05$ compared with control cells without LPS; $\# P<0.05$ compared with control cells with LPS. The data presented are one representative assessment of triplicate assays with similar results. 
shRNA vs. CON shRNA+LPS, $1.19 \pm 0.08$ vs. $0.72 \pm 0.04$, all $P<0.05)$. Figure $2 \mathrm{~B} \& \mathrm{C}$ illustrates increased apoptosis in response to LPS treatment (CON vector vs. CON vector+LPS, $4.24 \pm 0.28$ vs. $31.71 \pm 2.11$; CON shRNA vs. CON shRNA+LPS, $5.93 \pm 0.25$ vs. $32.59 \pm 2.05$, all $P<0.05)$. After TREM-1 vector or shRNA treatment, the TREM-1 shRNA+LPS group exhibited increased cell proliferation compared to the CON shRNA+LPS group $(0.90 \pm 0.06$ vs. $0.72 \pm 0.04$, $P<0.05)$, while the TREM-1 vector+LPS group exhibited decreased cell proliferation compared to the CON vector+LPS group $(0.58 \pm 0.08$ vs. $0.77 \pm 0.02$, $P<0.05)$. However, the ratio of apoptotic cells showed the opposite trend: the apoptosis rate in the TREM-1 shRNA+LPS group was lower than that in the CON shRNA+LPS group $(26.64 \pm 0.60$ vs. $32.59 \pm 2.05$, $P<0.05)$, while that in the TREM-1 vector+LPS group was higher than that in the CON vector+LPS group $(51.79 \pm 1.08$ vs. $31.71 \pm 2.11, P<0.05)$.

\section{Cytokine expression in HK-2 cells was regulated by TREM-1 after LPS treatment}

The cross-linking of TREM-1 was shown to stimulate cytokine secretion in monocytes and neutrophils. Thus, we investigated whether TREM-1 expression in HK-2 cells is related to the production of IL-1 $\beta$, TNFa and IL-6 after LPS treatment (Figure 3). As shown in Figure 3, sTREM-1 levels were increased after LPS treatment in all groups (CON vector vs. CON vector+LPS, $56.85 \pm 0.68$ vs. $292.13 \pm 3.40$; CON shRNA vs. CON shRNA+LPS, $53.12 \pm 1.97$ vs. 292.13 \pm 6.80 ; TREM-1 vector vs. TREM-1 vector+LPS, $57.38 \pm 1.06$ vs. $326.06 \pm 9.00$; TREM-1 shRNA vs. TREM-1 shRNA+LPS, $44.97 \pm 1.98 ;$ all $P<0.05)$. However, the TREM-1 vector+LPS group had a significantly higher sTREM-1 level than the CON vector+LPS group, while the TREM-1 shRNA+LPS group had a significantly lower sTREM-1 level than the CON shRNA+LPS group. The expression levels of other cytokines showed the same trend; IL-1 $\beta$ (CON vector vs. CON vector+LPS, $1.69 \pm 0.17$ vs. $2.47 \pm 0.17$; CON shRNA vs. CON shRNA+LPS, $1.91 \pm 0.15$ vs. 2.66 \pm 0.07 ; TREM-1 vector vs. TREM-1 vector+LPS, $2.04 \pm 0.06$ vs. $3.54 \pm 0.58$; TREM- 1 shRNA vs. TREM- 1 shRNA+LPS, $1.66 \pm 0.15$ vs. $2.07 \pm 0.04$; all $P<0.05$ ), TNF-a (CON vector vs. CON vector+LPS, $70.87 \pm 1.25$ vs. $92.40 \pm 4.02$; CON shRNA vs. CON shRNA+LPS, $74.77 \pm 3.94$ vs. $117.08 \pm 6.76$; TREM-1 vector vs. TREM-1 vector+LPS, $92.40 \pm 4.02$ vs. $156.43 \pm 10.07$; TREM-1 shRNA vs. TREM-1 shRNA+LPS, $70.91 \pm 2.20$ vs.
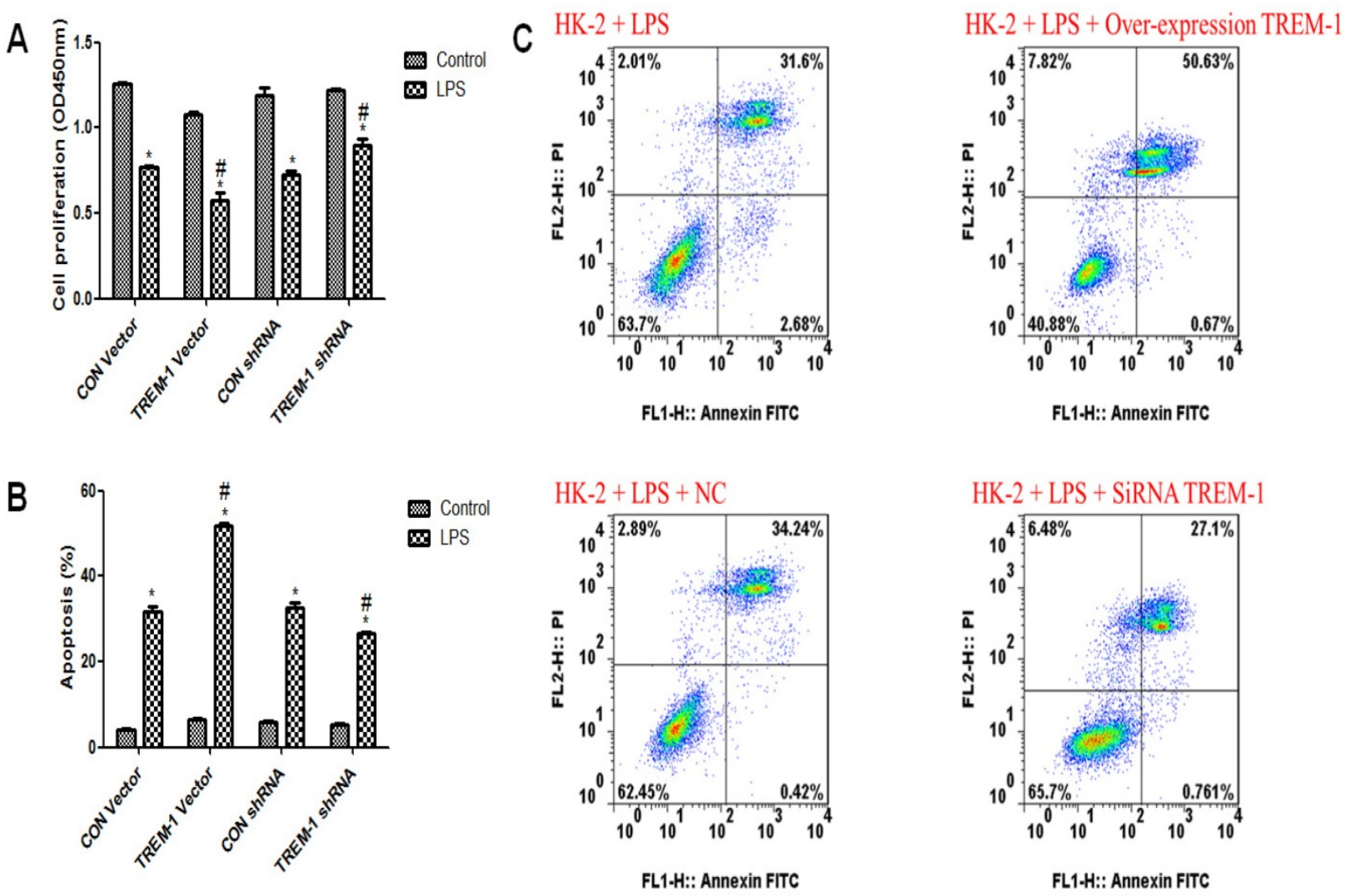

Figure 2. The roles of TREM-1 in modulating HK-2 cell proliferation and apoptosis in response to LPS. HK-2 cells were transfected with a control vector, TREM-1 vector, control shRNA, or TREM-1-specific shRNA as described in the Materials and Methods section. The cells were then cultured for $72 \mathrm{~h}$ with or without LPS (10 $\mu \mathrm{g} / \mathrm{mL})$. Panel A: Quantitative comparison of cell proliferation in cells with or without LPS exposure. Panel B: Quantitative comparison of cell apoptosis in cells with or without LPS exposure. Panel C: Flow cytometry of cell apoptosis in cells with or without LPS exposure. $* P<0.05$ compared with control cells without LPS; \# $P<0.05$ compared with control cells with LPS. The data presented are a representative assessment of triplicate assays with similar results. 


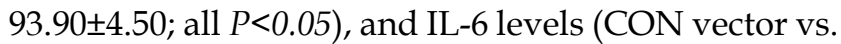
CON vector+LPS, $38.10 \pm 4.75$ vs. $62.65 \pm 4.50 ; C O N$ shRNA vs. CON shRNA+LPS, $41.68 \pm 2.52$ vs. $66.31 \pm 5.69$; TREM-1 vector vs. TREM-1 vector+LPS, $46.38 \pm 4.25$ vs. $73.97 \pm 7.71$; TREM- 1 shRNA vs. TREM-1 shRNA+LPS, $41.75 \pm 2.21$ vs. $57.23 \pm 3.81$; all $P<0.05$ ) were increased in the LPS-treated group compared with the control group without LPS. In addition, IL-1 $\beta$, TNF- $\alpha$, and IL-6 levels were significantly increased after TREM-1 vector transfection and significantly decreased after TREM-1-specific shRNA transfection.

\section{LPS-induced HK-2 cell apoptosis was mediated by TREM- 1}

Regarding the expression of apoptosisassociated proteins, as shown in Figure 4, Bcl-2 expression was decreased in cells stimulated with LPS in all groups (CON vector vs. CON vector+LPS, $0.51 \pm 0.02$ vs. $0.26 \pm 0.01$; CON shRNA vs. CON shRNA+LPS, $0.45 \pm 0.01$ vs. $0.21 \pm 0.01$; TREM- 1 vector vs. TREM-1 vector+LPS, $0.51 \pm 0.03$ vs. $0.20 \pm 0.02$; TREM-1 shRNA vs. TREM-1 shRNA+LPS, $0.72 \pm 0.08$ vs. $0.30 \pm 0.01$; all $P<0.05)$. However, the TREM-1 vector+LPS group had a significantly lower $\mathrm{Bcl}-2$ level than the CON vector+LPS group, while the TREM-1 shRNA+LPS group had a significantly higher Bcl-2 level than the CON shRNA+LPS group. In contrast, the expression patterns of Bax, cleaved caspase- 3 and cleaved caspase-9 differed. After LPS treatment, Bax (CON vector vs. CON vector+LPS, $0.74 \pm 0.04$ vs. $0.97 \pm 0.13$; CON shRNA vs. CON shRNA+LPS, $0.67 \pm 0.04$ vs. $1.18 \pm 0.12$; TREM-1 vector vs. TREM-1 vector+LPS, $0.89 \pm 0.08$ vs. $1.27 \pm 0.12$; TREM- 1 shRNA vs. TREM- 1 shRNA+LPS, $0.70 \pm 0.07$ vs. $0.94 \pm 0.18$; all $P<0.05)$, cleaved caspase-3 (CON vector vs. CON vector+LPS, $0.31 \pm 0.03$ vs. $1.30 \pm 0.10$; CON shRNA vs. CON shRNA+LPS, $0.28 \pm 0.0 .02$ vs. $1.57 \pm 0.12$; TREM-1 vector vs. TREM-1 vector+LPS, $0.50 \pm 0.19$ vs. 1.98 \pm 0.15 ; TREM-1 shRNA vs. TREM-1 shRNA+LPS, $0.16 \pm 0.01$ vs. $1.19 \pm 0.12$; all $P<0.05$ ) and cleaved caspase-9 (CON vector vs. CON vector+LPS, $0.17 \pm 0.04$ vs. $0.49 \pm 0.02$; CON shRNA vs. CON shRNA+LPS, $0.17 \pm 0.03$ vs. $0.49 \pm 0.05$; TREM- 1 vector vs. TREM-1 vector+LPS, $0.29 \pm 0.03$ vs. $0.73 \pm 0.03$; TREM-1 shRNA vs. TREM-1 shRNA+LPS, $0.12 \pm 0.03$ vs. $0.38 \pm 0.02$; all $P<0.05$ ) levels were significantly increased. However, the TREM-1 vector+LPS group had significantly higher Bax, cleaved caspase- 3 and cleaved caspase-9 levels than the CON vector+LPS group, while the TREM-1 shRNA+LPS group had significantly lower Bax, cleaved caspase-3 and cleaved caspase-9 levels than the CON shRNA+LPS group.
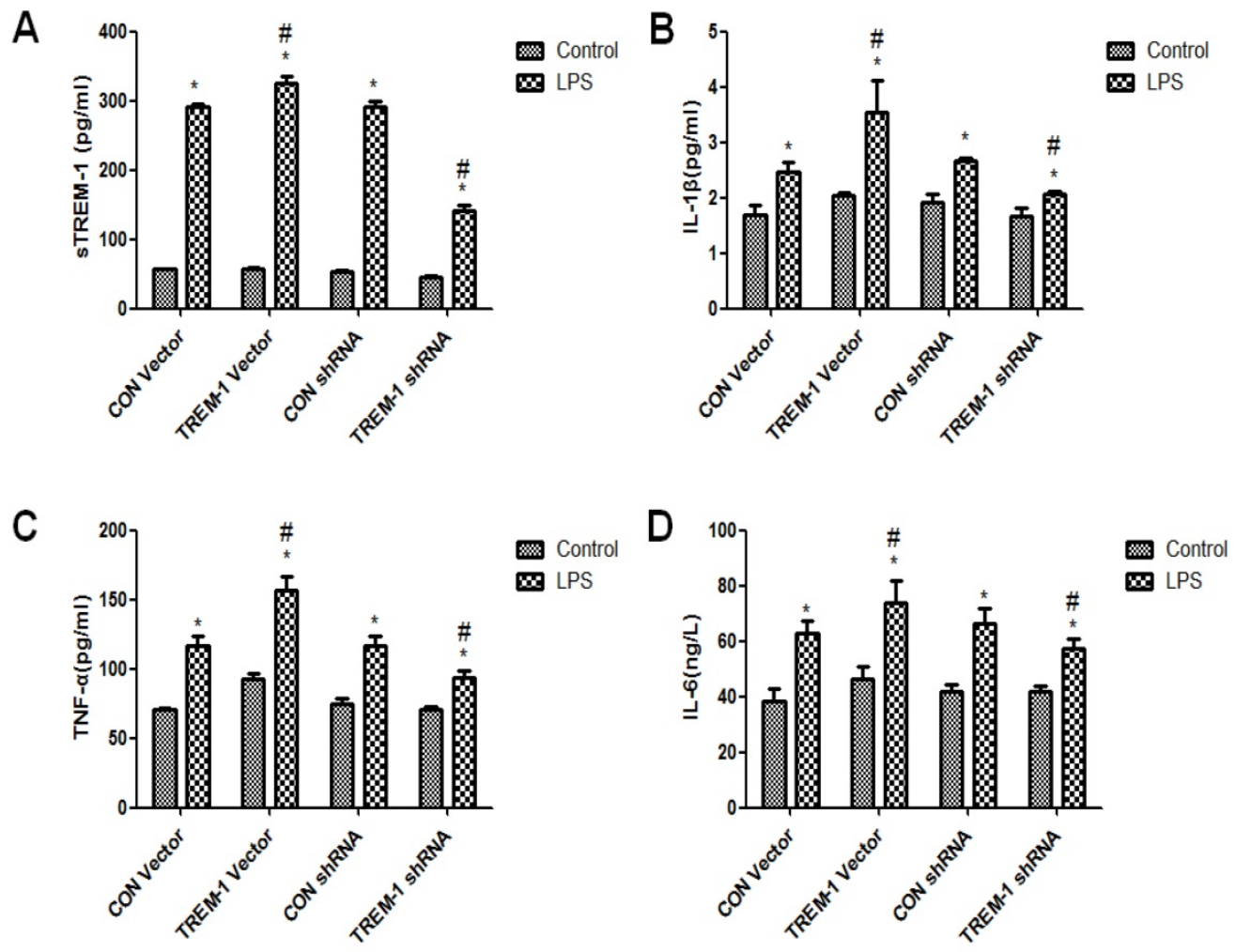

Figure 3. Inflammation and cytokine levels in supernatants of HK-2 cells treated with a TREM-1 vector or shRNA with or without LPS. The concentrations of sTREM-1 (panel A), IL-1 1 (panel B), TNFa (panel C), and IL-6 (panel D) were measured. HK-2 cells were transfected with a control vector, plasmid for TREM-1 expression, control siRNA or TREM-1-specific siRNA as described in the Materials and Methods section. $* P<0.05$ compared with control cells without LPS; \# $P<0.05$ compared with control cells with LPS. The data presented are a representative assessment of triplicate assays with similar results. 
A

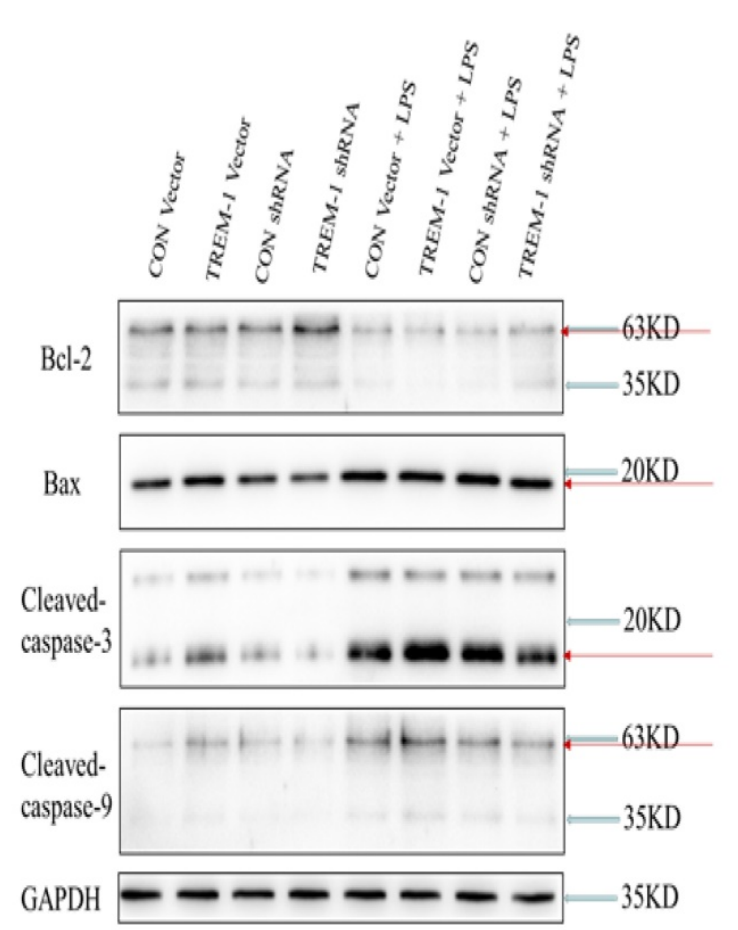

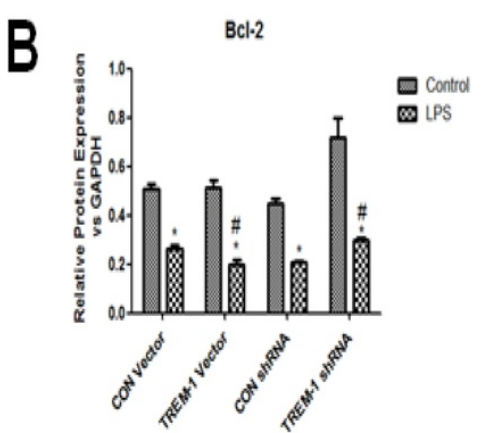
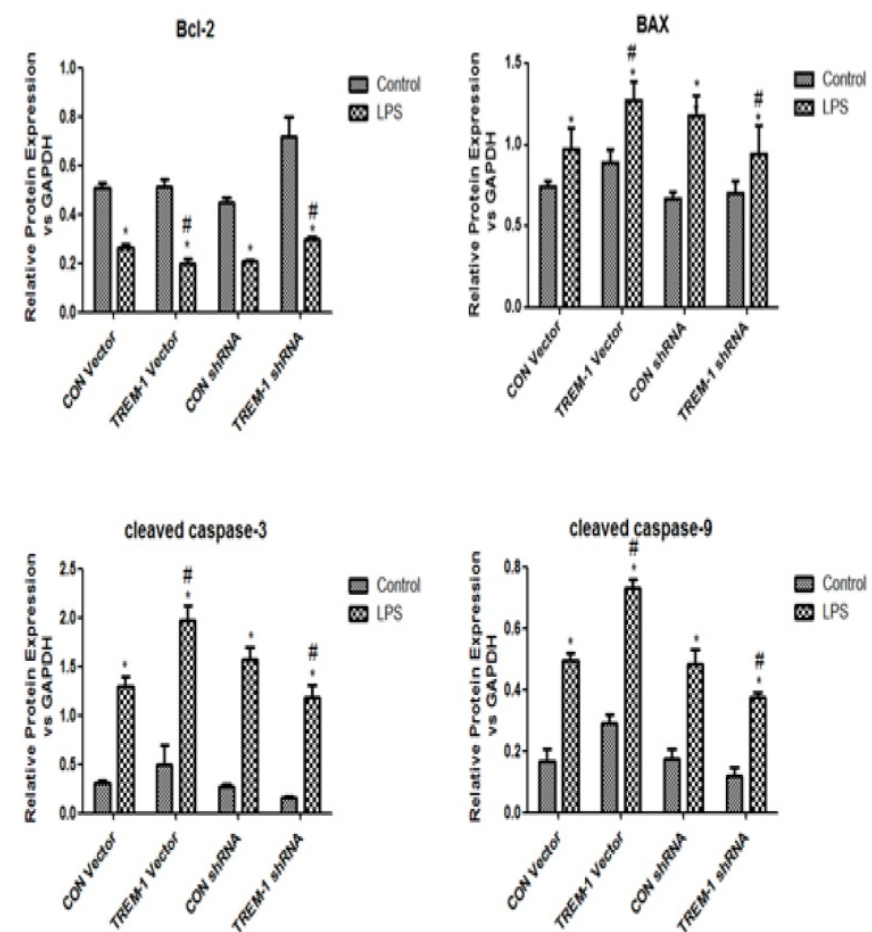

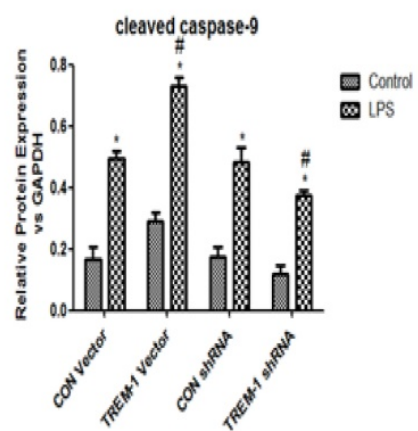

Figure 4. LPS-induced HK-2 cell apoptosis was mediated by TREM-1. HK-2 cells were transfected with a plasmid containing a negative control vector/TREM-1 gene or negative control shRNA/TREM-1-specific shRNA, followed by exposure to LPS $(10 \mu \mathrm{g} / \mathrm{mL})$. Bcl-2, Bax, cleaved caspase- 3 , and cleaved caspase-9 were assessed by immunoblotting as described in the Materials and Methods section. Panel A: Representative images showing the results of immunoblotting. Panel B: Average of three separate immunoblots for Bcl-2, Bax, cleaved caspase-3, and cleaved caspase-9. Vertical axis: ratio to control; horizontal axis: cells transfected with plasmids containing negative control vector/TREM-1 gene or negative control shRNA/TREM-1 shRNA. $* P<0.05$.

\section{LPS-induced HK-2 cell autophagy was mediated by TREM-1}

Autophagy was further assessed by western blot analysis of expression of the autophagy-related genes Beclin1, ATG5, LC3B-II and p62 in cells treated with or without LPS, as shown in Figure 5. The expression of Beclin1, ATG5, and LC3B-II was increased in the cells stimulated with LPS in all groups (Beclin: CON vector vs. CON vector+LPS, $0.10 \pm 0.01$ vs. $0.22 \pm 0.01$; CON shRNA vs. CON shRNA+LPS, $0.09 \pm 0.01$ vs. $0.22 \pm 0.01$; TREM-1 vector vs. TREM-1 vector+LPS, $0.14 \pm 0.01$ vs. $0.16 \pm 0.02$; TREM- 1 shRNA vs. TREM- 1 shRNA+LPS, $0.07 \pm 0.01$ vs. $0.29 \pm 0.01$; all $P<0.05$; Atg 5 : $\mathrm{CON}$ vector vs. CON vector+LPS, $0.81 \pm 0.02$ vs. $0.93 \pm 0.04 ;$ CON shRNA vs. CON shRNA+LPS, $0.55 \pm 0.06$ vs. $1.01 \pm 0.05$; TREM-1 vector vs. TREM-1 vector+LPS, $0.94 \pm 0.05$ vs. $1.14 \pm 0.09$; TREM- 1 shRNA vs. TREM- 1 shRNA+LPS, $0.57 \pm 0.07$ vs. $1.37 \pm 0.05$; all $P<0.05$; LC3B: CON vector vs. CON vector+LPS, $0.42 \pm 0.02$ vs. $0.49 \pm 0.04 ; \mathrm{CON}$ shRNA vs. CON shRNA+LPS, $0.16 \pm 0.01$ vs. $0.58 \pm 0.04$; TREM- 1 vector vs. TREM-1 vector+LPS, $0.44 \pm 0.02$ vs. $0.66 \pm 0.08$; TREM-1 shRNA vs. TREM-1 shRNA+LPS, $0.19 \pm 0.01$ vs. $0.86 \pm 0.04$; all $P<0.05$ ). However, p62 expression followed the opposite trend (CON vector vs. $\mathrm{CON}$ vector+LPS, $1.30 \pm 0.05$ vs. $0.41 \pm 0.05$; CON shRNA vs. CON shRNA+LPS, $1.07 \pm 0.03$ vs. $0.47 \pm 0.03$; TREM- 1 vector vs. TREM-1 vector+LPS, $0.36 \pm 0.05$ vs. $0.90 \pm 0.07$; TREM-1 shRNA vs. TREM-1 shRNA+LPS, $1.30 \pm 0.02$ vs. $0.34 \pm 0.01$; all $P<0.05$ ). Densitometric analysis of the protein bands revealed that as expected, overexpression of TREM-1 in the LPS-treated group induced the expression of autophagy-related genes and had the opposite effect on the degradation-related protein p62. In LPS-treated cells, the expression of autophagy-related genes was further upregulated, and p62 expression was further downregulated compared with that in control cells.

\section{The transcriptional level of NF-KB was regulated by TREM-1 in HK-2 cells}

We investigated the role of transcription factors in the induction of TREM-1 expression (Figure 6). Similar to the results for the apoptosis- and autophagy-related proteins, the expression of P-p65, p65, P-IкBa, and IкBa in all the groups was increased after LPS treatment (P-p65/p65: CON vector vs. CON vector+LPS, $0.12 \pm 0.01$ vs. $0.48 \pm 0.05$; CON shRNA vs. CON shRNA+LPS, $0.26 \pm 0.01$ vs. $0.52 \pm 0.03$; TREM- 1 vector vs. TREM-1 vector+LPS, $0.31 \pm 0.05$ vs. 0.78 \pm 0.14 ; TREM-1 shRNA vs. TREM-1 shRNA+LPS, $0.18 \pm 0.01$ vs. $0.38 \pm 0.03$; all $P<0.05 ; \mathrm{P}-\mathrm{I \kappa Ba}, / \mathrm{I \kappa Ba}$ : CON vector vs. CON vector+LPS, $0.28 \pm 0.05$ vs. $0.946 \pm 0.02$; CON shRNA vs. CON shRNA+LPS, $0.24 \pm 0.01$ vs. $0.48 \pm 0.03$; TREM-1 vector vs. TREM-1 vector+LPS, 
$0.38 \pm 0.06$ vs. $0.63 \pm 0.07$; TREM- 1 shRNA vs. TREM- 1 shRNA+LPS, $0.25 \pm 0.02$ vs. $0.40 \pm 0.01$; all $P<0.05)$. The TREM-1 vector+LPS group had higher P-p65, p65, P-IKBa, and IкBa levels than the CON vector+LPS group. However, the TREM-1 shRNA+LPS group had lower P-p65, p65, Р-ІкBa, and ІкBa levels than the CON shRNA+LPS group.
A

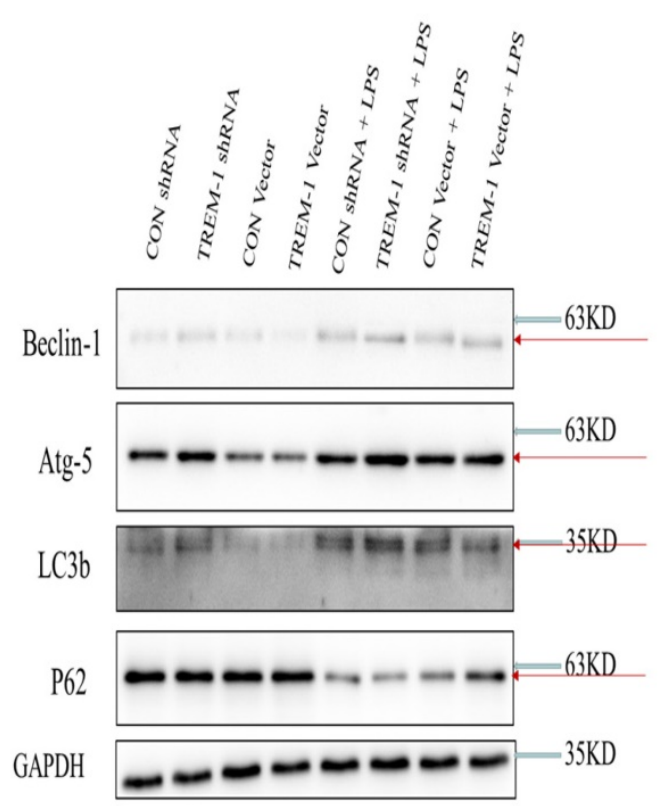

B
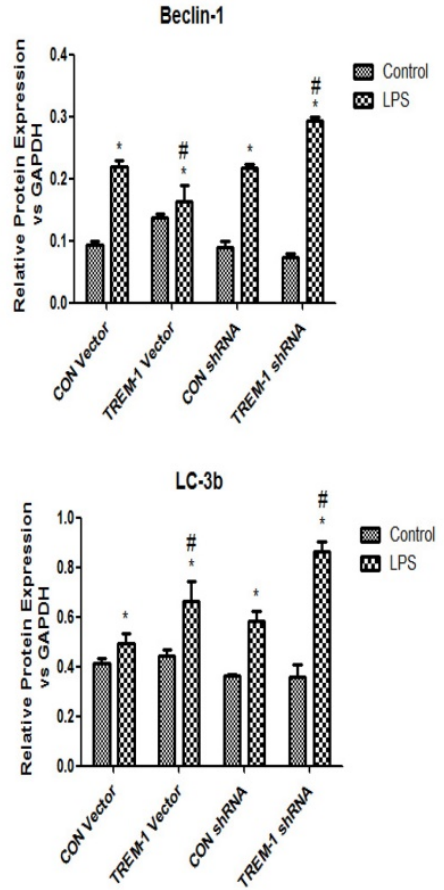

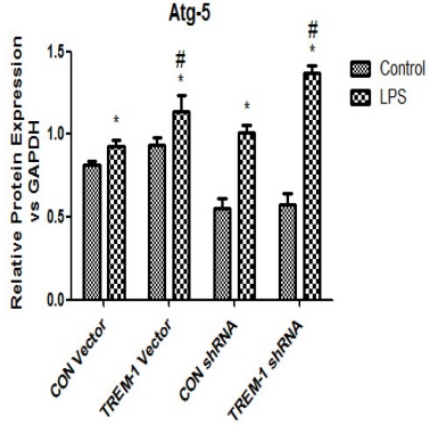

P62

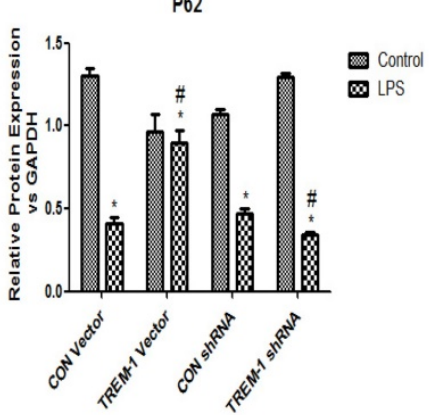

Figure 5. LPS-induced HK-2 cell autophagy was mediated by TREM-1. HK-2 cells were transfected with a plasmid containing a negative control vector/TREM-1 gene or negative control shRNA/TREM-1-specific shRNA, followed by exposure to LPS $(10 \mu \mathrm{g} / \mathrm{mL})$. Beclin-1, Atg-5, LC-3b, and p62 were assessed by immunoblotting as described in the Materials and Methods section. Panel A: Representative images of immunoblots. Panel B: Averages of three separate immunoblots for Beclin-1, Atg-5, LC-3b, and p62. Vertical axis: ratio to control; horizontal axis: cells transfected with plasmids containing a negative control vector/TREM-1 gene or negative control shRNA/TREM-1-specific shRNA. $* P<0.05$.

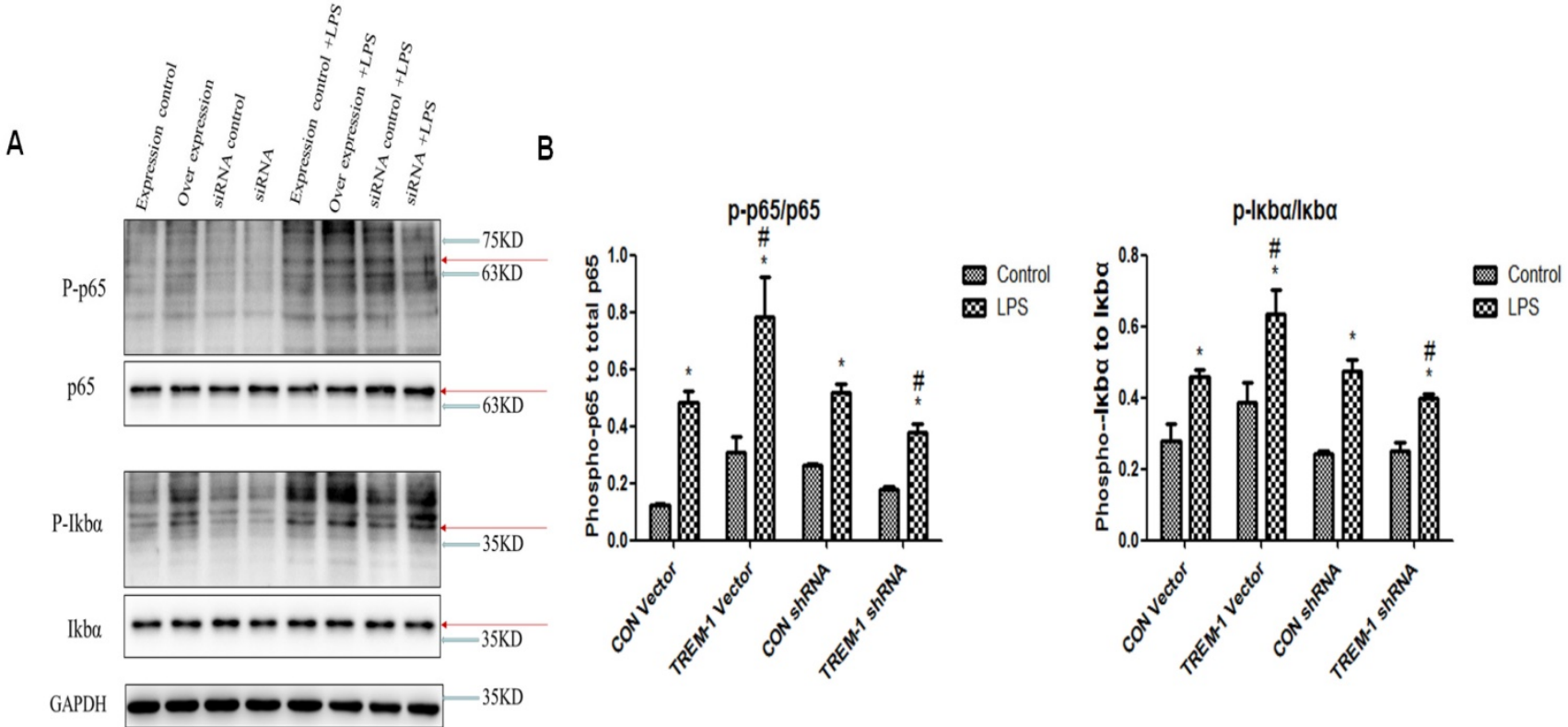

Figure 6. The NF-KB signaling pathway in LPS-treated HK-2 cells was regulated by TREM-1. HK-2 cells were transfected with plasmid containing a negative control vector/TREM-1 gene or negative control shRNA/TREM-1-specific shRNA, followed by exposure to LPS $(10 \mu g / \mathrm{mL})$. P-p65, p65, P-IKBa, and IKBa were assessed by immunoblotting as described in the Materials and Methods section. Panel A: Representative images of immunoblots. Panel B: Average of three separate immunoblots for $\mathrm{P}-\mathrm{p} 65 / \mathrm{p} 65$ and P-IKBa/lKBa. Vertical axis: ratio to control; horizontal axis: cells transfected with plasmids containing negative control vector/TREM-1 gene or negative control ShRNA/TREM-1 shRNA. $* P<0.05$. 


\section{Discussion}

In the present work, we examined the potential roles of TREM-1 in apoptosis and autophagy in HK-2 cells treated with LPS. Notably, expression of the antiapoptotic gene $\mathrm{Bcl}-2$ was downregulated in the TREM-1 overexpression group, while that of the proapoptotic genes Bax, cleaved caspase-3 and cleaved caspase- 9 was upregulated. Overexpression of TREM-1 downregulated expression of the autophagy-related genes Beclin-1, Atg-5 and LC $3 \mathrm{~b}$ and upregulated the gene expression of p62, which inhibits autophagy. Our data further suggested that the changes in apoptosis and autophagy induced by LPS were associated with TREM1-NF-kB inflammation-dependent signaling.

TREM-1 could not only mediate sepsis-related kidney damage but also contribute to inflammation in IgA nephropathy, which has been detected by urinalysis [21]. sTREM-1 levels were also shown to be significantly increased in hemodialysis patients and positively correlated with CRP and TNF-a levels [22]. In our study, we found that the levels of IL-1 $\beta$, TNFa and IL- 6 were increased after TREM- 1 overexpression but decreased after TREM-1 downregulation. A cell proliferation assay showed enhanced cell proliferation after TREM-1-specific siRNA intervention. Our findings are consistent with in vitro studies that demonstrated that TREM-1 blockade by the inhibitory peptide LP17 prolonged the survival of rats that underwent cecum ligation and puncture (CLP) and attenuated systematic inflammatory responses [23]. However, some studies have reached the opposite conclusion, instead showing that TREM-1 can attenuate the inflammatory response and reduce tissue damage [24, 25]. The mechanism behind this discrepancy remains to be explained.

Our study showed that cell apoptosis was increased after LPS intervention regardless of TREM-1 overexpression or downregulation. From our results, although the level of apoptosis after TREM-1 inhibition was not as high as that after TREM-1 activation, there was no significant difference in the degree of apoptosis. Theoretically, TREM-1 overexpression enhances the increase in mitochondrial ROS caused by LPS, leading to mitochondrial damage, excessive inflammation and apoptosis, while TREM-1 downregulation has the opposite result. In some studies, TREM-1-knockout macrophages exhibited increased apoptosis in response to LPS [26]. TREM-1 was also shown to protect monocytes from apoptosis through the regulation of myeloid cell leukemia-1 [27]. These results showed that different cell types, concentrations of LPS for intervention and durations may influence the role of TREM-1 in apoptosis. Most importantly, autophagy may participate in this biological process. After TREM-1 was inhibited, the level of autophagy in cells was further increased. This result may have been because the regulation of autophagy caused further damage to cells through apoptosis. TREM-1 was reported to modulate autophagic activity and endoplasmic reticulum (ER) stress in colitis in mice [28]. Furthermore, TREM-1 in human monocytes/macrophages and epithelial cells was shown in some studies to be regulated by 1a,25-dihydroxyvitamin D3 via mTOR signaling [29]. Therefore, we have inferred that TREM-1 may participate in the autophagic process. TREM-1 deficiency could attenuate disease severity and organ injury without affecting pathogen clearance [30].

The NF-kB pathway is an important inflammatory signaling pathway. A number of previous studies have suggested that TREM- 1 plays a key role upstream of this signal. TREM-1 was found to play a critical role in osteoarthritis development through the regulation of NF- $\mathrm{kB}$ signaling [15]. The inhibition of $\mathrm{sEH}$ by 1-trifluoromethoxyphenyl3-(1-propionylpiperidin-4-yl) urea (TPPU) suppressed LPS-induced TREM-1 expression and inflammation by inhibiting NF- $\mathrm{kB}$ activation in murine macrophages [31]. Wang et al [32] suggested that aggravation of ventilation-induced lung injury (VILI) by TREM-1 in mice was associated with TLR4-MyD88-NF-kB-dependent signaling. $\mathrm{Zi}$ et al [33] demonstrated that high sTREM-1 levels were independent predictors of increased endothelial microparticle levels on admission and associated with increased risks for mortality and major adverse cardiovascular events in acute myocardial infarction patients. It was further reported that Sirt6-induced autophagy restricted TREM-1-mediated pyroptosis in ox-LDL-treated endothelial cells. Additionally, TREM-1 and NLRP3 could regulate the inflammatory response of lung macrophages and even improve lung injury [34]. TREM-1 was found to aggravate inflammation in acute lung injury (ALI) by activating the NLRP3 inflammasome [35]. We found that TREM-1 could regulate NF-KB signaling to influence the processes of apoptosis and autophagy. However, we did not attempt to block NF-KB to evaluate its effect on autophagy. The mechanisms by which NF-kB regulates autophagy and those by which TREM-1 mediates pyroptosis and autophagy remain to be further studied.

\section{Conclusion}

TREM-1 increased the apoptosis of renal TECs and reduced autophagy by mediating LPS intervention. Imbalance between apoptosis and autophagy is one of the core mechanisms of kidney 
injury after sepsis. Blockade of TREM-1 resulted in decreased apoptosis and increased autophagy, which may have been due to an increase in inflammatory damage caused by modulation of the NF-kB pathway. The TREM-1 receptor may indirectly affect mitochondria through its interactions with ligands, thereby enhancing inflammation, or directly affect mitochondria through signal transduction pathways. TREM-1 may be a key target for intervention in renal function damage, but further work is needed in the future.

\section{Abbreviations}

AKI, acute kidney injury; TREM-1, triggering receptor expressed by myeloid cells; HK-2 cells, human kidney-2 cells; SA-AKI, sepsis-associated acute kidney injury; LPS, lipopolysaccharide; VILI, ventilation-induced lung injury.

\section{Acknowledgements}

This work was supported by the Beijing Municipal Natural Science Foundation (No. 7192163 to Longxiang $\mathrm{Su}$ ); the Beijing Nova Program from Beijing Municipal Science \& Technology Commission (No. Z201100006820126 to Longxiang Su); the Open Project of the State Key Laboratory of Kidney Diseases, Chinese People's Liberation Army General Hospital (KF-19-08 to Longxiang Su); the National Science Foundation for Young Scientists of China (No. 81901935 to Pan Pan); the Capital Foundation of Medical Development (No. 2016-1-5015 to Lixin Xie); and the China National Key Reseach Program (Grant no. 2018ZX09201013 to Lixin Xie). The funders had no role in the study design, data collection and analysis, decision to publish, or preparation of the manuscript.

\section{Competing Interests}

The authors have declared that no competing interest exists.

\section{References}

1. Singer M, Deutschman CS, Seymour CW, Shankar-Hari M, Annane D, Bauer M, et al. The Third International Consensus Definitions for Sepsis and Septic Shock (Sepsis-3). JAMA. 2016; 315: 801-10.

2. Pool R, Gomez H, Kellum JA. Mechanisms of Organ Dysfunction in Sepsis. Crit Care Clin. 2018; 34: 63-80.

3. Poston JT, Koyner JL. Sepsis associated acute kidney injury. BMJ. 2019; 364: k4891.

4. Otero RM, Nguyen HB, Huang DT, Gaieski DF, Goyal M, Gunnerson KJ, et al. Early goal-directed therapy in severe sepsis and septic shock revisited: concepts, controversies, and contemporary findings. Chest. 2006; 130: 1579-95.

5. Su L, Han B, Liu C, Liang L, Jiang Z, Deng J, et al. Value of soluble TREM-1, procalcitonin, and C-reactive protein serum levels as biomarkers for detecting bacteremia among sepsis patients with new fever in intensive care units: a prospective cohort study. BMC Infect Dis. 2012; 12: 157

6. Su L, Feng L, Song Q, Kang H, Zhang X, Liang Z, et al. Diagnostic value of dynamics serum sCD163, sTREM-1, PCT, and CRP in differentiating sepsis, severity assessment, and prognostic prediction. Mediators Inflamm. 2013; 2013: 969875.
7. Su L, Xie L, Liu D. Urine sTREM-1 may be a valuable biomarker in diagnosis and prognosis of sepsis-associated acute kidney injury. Crit Care. 2015; 19: 281.

8. Su LX, Feng L, Zhang J, Xiao YJ, Jia YH, Yan P, et al. Diagnostic value of urine sTREM-1 for sepsis and relevant acute kidney injuries: a prospective study. Crit Care. 2011; 15: R250.

9. Gomez-Pina V, Soares-Schanoski A, Rodriguez-Rojas A, Del Fresno C, Garcia F, Vallejo-Cremades MT, et al. Metalloproteinases shed TREM-1 ectodomain from lipopolysaccharide-stimulated human monocytes. J Immunol. 2007; 179: 4065-73.

10. Ford JW, McVicar DW. TREM and TREM-like receptors in inflammation and disease. Curr Opin Immunol. 2009; 21: 38-46.

11. Bouchon A, Dietrich J, Colonna M. Cutting edge: inflammatory responses can be triggered by TREM-1, a novel receptor expressed on neutrophils and monocytes. J Immunol. 2000; 164: 4991-5.

12. Bouchon A, Facchetti F, Weigand MA, Colonna M. TREM-1 amplifies inflammation and is a crucial mediator of septic shock. Nature. 2001;410: 1103-7.

13. Schenk M, Bouchon A, Seibold F, Mueller C. TREM-1--expressing intestinal macrophages crucially amplify chronic inflammation in experimental colitis and inflammatory bowel diseases. J Clin Invest. 2007; 117: 3097-106.

14. Colonna M, Facchetti F. TREM-1 (triggering receptor expressed on myeloid cells): a new player in acute inflammatory responses. J Infect Dis. 2003; 187 Suppl 2: S397-401.

15. Tang J, Dong Q. Knockdown of TREM-1 suppresses IL-1beta-induced chondrocyte injury via inhibiting the NF-kappaB pathway. Biochem Biophys Res Commun. 2017; 482: 1240-5.

16. Kinsey GR, Li L, Okusa MD. Inflammation in acute kidney injury. Nephron Exp Nephrol. 2008; 109: e102-7.

17. Akcay A, Nguyen Q, Edelstein CL. Mediators of inflammation in acute kidney injury. Mediators Inflamm. 2009; 2009: 137072.

18. Bonavia A, Singbartl K. A review of the role of immune cells in acute kidney injury. Pediatr Nephrol. 2018; 33: 1629-39.

19. Tammaro A, Scantlebery AML, Rampanelli E, Borrelli C, Claessen N, Butter LM, et al. TREM1/3 Deficiency Impairs Tissue Repair After Acute Kidney Injury and Mitochondrial Metabolic Flexibility in Tubular Epithelial Cells. Front Immunol. 2019; 10: 1469.

20. Dai X, Zeng Z, Fu C, Zhang S, Cai Y, Chen Z. Diagnostic value of neutrophil gelatinase-associated lipocalin, cystatin $C$, and soluble triggering receptor expressed on myeloid cells- 1 in critically ill patients with sepsis-associated acute kidney injury. Crit Care. 2015; 19: 223.

21. Zhao YF, Zhu L, Liu LJ, Shi SF, Lv JC, Zhang H. TREM-1 Contributes to Inflammation in IgA Nephropathy. Kidney Dis (Basel). 2018; 4: 29-36.

22. Essa ES, Elzorkany KM. sTREM-1 in patients with chronic kidney disease on hemodialysis. APMIS. 2015; 123: 969-74.

23. Shi X, Zhang Y, Wang H, Zeng S. Effect of Triggering Receptor Expressed on Myeloid Cells 1 (TREM-1) Blockade in Rats with Cecal Ligation and Puncture (CLP)-Induced Sepsis. Med Sci Monit. 2017; 23: 5049-55.

24. Syed MA, Shah D, Das P, Andersson S, Pryhuber G, Bhandari V. TREM-1 Attenuates RIPK3-mediated Necroptosis in Hyperoxia-induced Lung Injury in Neonatal Mice. Am J Respir Cell Mol Biol. 2019; 60: 308-22.

25. Tammaro A, Kers J, Emal D, Stroo I, Teske GJD, Butter LM, et al. Effect of TREM-1 blockade and single nucleotide variants in experimental renal injury and kidney transplantation. Sci Rep. 2016; 6: 38275.

26. Yuan Z, Syed MA, Panchal D, Joo M, Colonna M, Brantly M, et al. Triggering receptor expressed on myeloid cells 1 (TREM-1)-mediated Bcl-2 induction prolongs macrophage survival. J Biol Chem. 2014; 289: 15118-29.

27. Cai M, Chen Q, Chen C, Liu X, Hou J, Zeng C, et al. Activation of triggering receptor expressed on myeloid cells-1 protects monocyte from apoptosis through regulation of myeloid cell leukemia-1. Anesthesiology. 2013; 118: 1140-9.

28. Kokten T, Gibot S, Lepage P, D'Alessio S, Hablot J, Ndiaye NC, et al. TREM-1 Inhibition Restores Impaired Autophagy Activity and Reduces Colitis in Mice. J Crohns Colitis. 2018; 12: 230-44.

29. Lee B, Kwon E, Kim Y, Kim JH, Son SW, Lee JK, et al. 1alpha,25-Dihydroxyvitamin D3 upregulates HIF-1 and TREM-1 via mTOR signaling. Immunol Lett. 2015; 163: 14-21.

30. Weber B, Schuster S, Zysset D, Rihs S, Dickgreber N, Schurch C, et al. TREM-1 deficiency can attenuate disease severity without affecting pathogen clearance. PLoS Pathog. 2014; 10: e1003900.

31. Dong L, Zhou Y, Zhu ZQ, Liu T, Duan JX, Zhang J, et al. Soluble Epoxide Hydrolase Inhibitor Suppresses the Expression of Triggering Receptor Expressed on Myeloid Cells-1 by Inhibiting NF-kB Activation in Murine Macrophage. Inflammation. 2017; 40: 13-20.

32. Wang X, Luo B, Lu Y, Pang D, Zheng J, Mo J, et al. The triggering receptor expressed by myeloid cells-1 activates 
TLR4-MyD88-NF-kappaB-dependent signaling to aggravate ventilation-induced lung inflammation and injury in mice. Cell Tissue Res. 2018; 374: 137-48.

33. Zi Y, Yi-An Y, Bing J, Yan L, Jing T, Chun-Yu G, et al. Sirt6-induced autophagy restricted TREM-1-mediated pyroptosis in ox-LDL-treated endothelial cells: relevance to prognostication of patients with acute myocardial infarction. Cell Death Discov. 2019; 5: 88.

34. Yadav N, Chandra H. Suppression of inflammatory and infection responses in lung macrophages by eucalyptus oil and its constituent 1,8-cineole: Role of pattern recognition receptors TREM-1 and NLRP3, the MAP kinase regulator MKP-1, and NFkappaB. PLoS One. 2017; 12: e0188232.

35. Liu T, Zhou Y, Li P, Duan JX, Liu YP, Sun GY, et al. Blocking triggering receptor expressed on myeloid cells-1 attenuates lipopolysaccharide-induced acute lung injury via inhibiting NLRP3 inflammasome activation. Sci Rep. 2016; 6: 39473. 